\title{
Clinical Neurophysiology in Alzheimer's Disease
}

\author{
Florinda Ferreri, ${ }^{1,2}$ Sara Määttä, ${ }^{1}$ Fabrizio Vecchio, ${ }^{3}$ Giuseppe Curcio, ${ }^{4}$ \\ and Fabio Ferrarelli ${ }^{5}$ \\ ${ }^{1}$ Department of Clinical Neurophysiology, School of Medicine, University of Eastern Finland, 70211 Kuopio, Finland \\ ${ }^{2}$ Department of Neurology, Campus Bio-medico University of Rome, 00128 Rome, Italy \\ ${ }^{3}$ AFaR, Department of Neuroscience, Fatebenefratelli Hospital, Isola Tiberina, 00186 Rome, Italy \\ ${ }^{4}$ Department of Health Sciences, University of L'Aquila, 67010 Coppito, Italy \\ ${ }^{5}$ Department of Psychiatry, School of Medicine and Public Health, University of Wisconsin-Madison, Madison, WI 53719, USA
}

Correspondence should be addressed to Florinda Ferreri, f.ferreri@unicampus.it

Received 9 June 2011; Accepted 9 June 2011

Copyright (c) 2011 Florinda Ferreri et al. This is an open access article distributed under the Creative Commons Attribution License, which permits unrestricted use, distribution, and reproduction in any medium, provided the original work is properly cited.

The pathophysiological mechanisms underlying normal aging and neurodegenerative disorders such as Alzheimer's disease (AD) have yet to be fully established. Early recognition of mild cognitive impairment (MCI) and $\mathrm{AD}$ requests the identification of biomarkers capable of distinguishing individuals with prodromes from healthy aging adults. Physiological brain aging is characterized by a loss of synaptic contacts and neuronal apoptosis even though neural redundancy as well as functional and structural plastic remodelling of brain networking promotes maintenance of brain activity in healthy elderly for everyday life. It is, then, important to implement techniques that are able to measure changes in normal aging brain and to discriminate them from neurodegenerative processes. As oscillatory electromagnetic brain activity is a hallmark of neuronal network function in various brain regions, an integrated approach utilizing modern neurophysiological techniques, including electroencephalography (EEG), event-related potentials (ERPs), and transcranial magnetic stimulation (TMS), together with biological markers and structural and functional imaging are promising for large-scale, affordable, and noninvasive evaluation of at-risk populations both at a group- and probably also at single-subject level.

This special issue contains a series of cutting-edge articles that provides innovative information and deal with the broad issue of the role of neurophysiology for the assessment of normal aging and dementia. Of necessity, these articles focus on selected topics but the mixture of novel contributions as well as review papers on EEG, TMS and ERP provide an overview and an insight into current areas of debate.

The first review of this special issue by G. Rodriguez et al., addresses an overview on the usefulness to study brain functional networks in the attempt to find noninvasive biomarkers of dementia; the second one, by R. Lizio et al., focuses on the role of modern EEG and on the possibility of combining its use together with biological and neuropsychological markers and structural and functional imaging for a low-cost, noninvasive, and widely available assessment of groups of individuals at-risk. Following these reviews, new EEG data are presented in the papers written by J. Dauwels et al. and F. Vecchio and C. Babiloni, dealing with the slowing and loss of complexity of EEG in $\mathrm{AD}$ patients and with the usefulness of EEG direction information flow in both $\mathrm{AD}$ and MCI patients, respectively. The subsequent three papers address EEG methodological issues, focusing on aspects helpful to better diagnose the AD: L.R. Trambaiolli et al. discuss in depth the possibility that EEG montage can influence $\mathrm{AD}$ diagnosis, F.B. Vialatte et al. provide suggestions on procedures to make EEG more specific in diagnosis, and K. van der Hiele et al. discuss the potential usefulness of electromyography in supporting the diagnosis of the disease. Then two review papers by F. Vecchio and S. Määttä and by A. Guerra et al., focus, respectively on the possibility to use auditory ERPs as well as TMS in complementing $\mathrm{AD}$ diagnosis, staging, and followup. The tenth study, by P. Julkunen et al., combines transcranial 
magnetic stimulation and electroencephalography (TMSEEG) to assess the severity of AD. Finally, two case reports are presented, in which neurophysiology plays a central role in supporting the diagnosis of dementia with particular attention to corticobasal loss of functionality (F. Mastrolilli et al.) and possible cooccurrence of nonconvulsive seizures and dementia (C. Campana et al.). As a corollary, the last contribution by L. Valeriani reports some considerations on the actual need in the management of $\mathrm{AD}$ patients in emergency departments.

Florinda Ferreri Sara Määttä

Fabrizio Vecchio

Giuseppe Curcio

Fabio Ferrarelli 


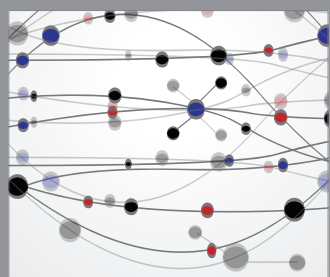

The Scientific World Journal
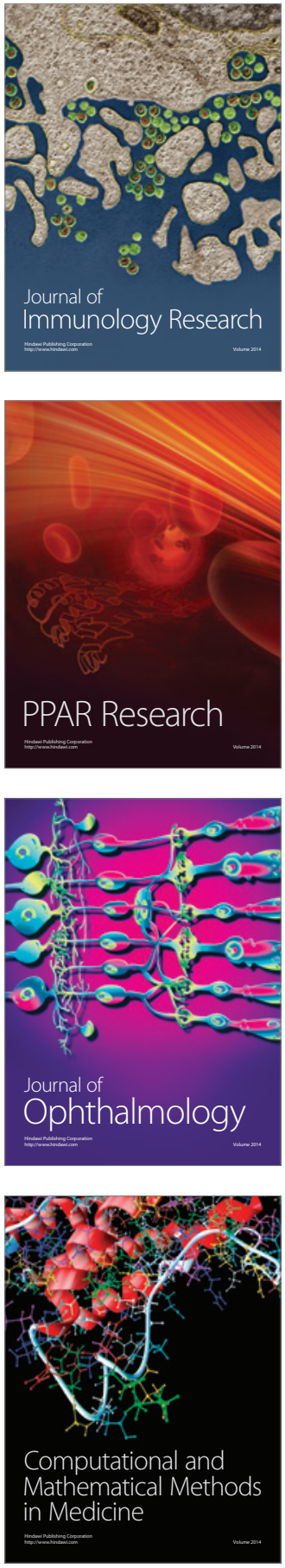

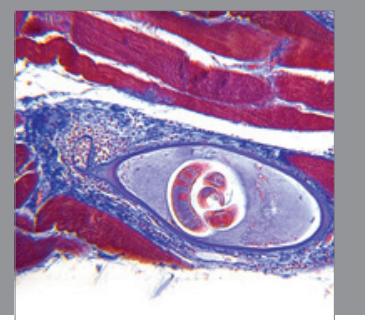

Gastroenterology

Research and Practice
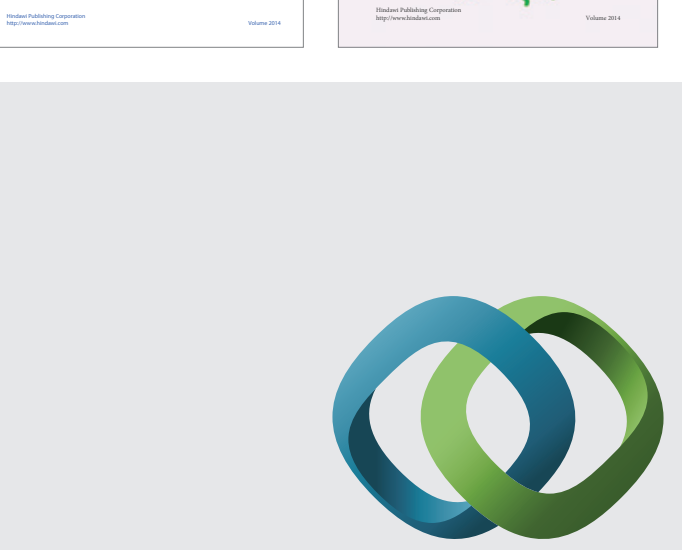

\section{Hindawi}

Submit your manuscripts at

http://www.hindawi.com
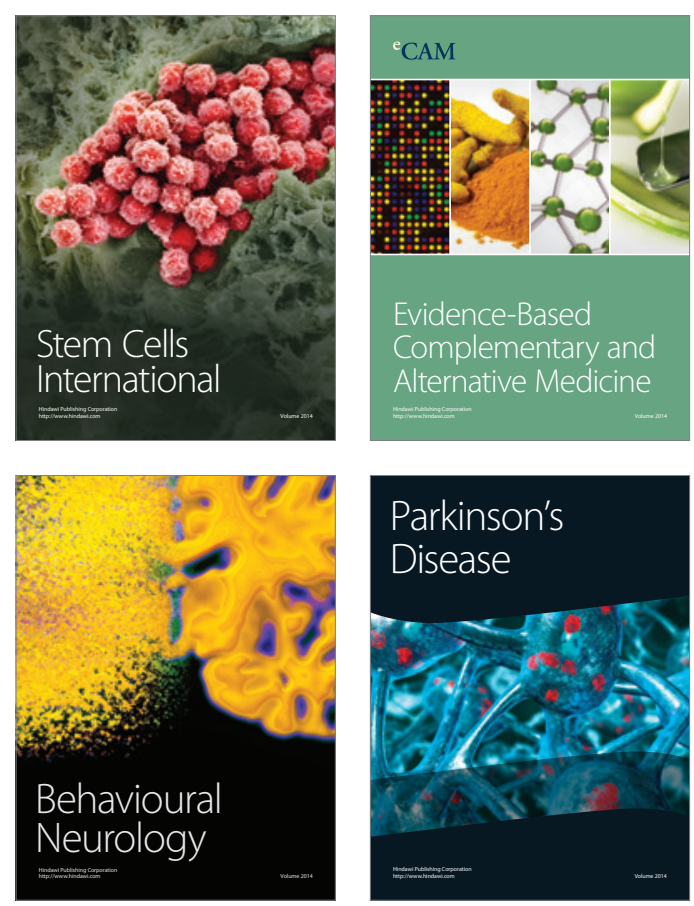

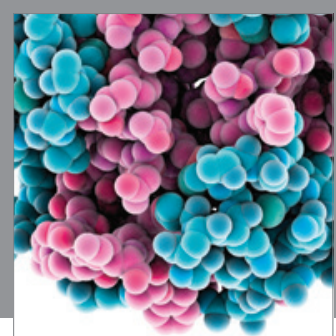

Journal of
Diabetes Research

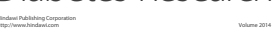

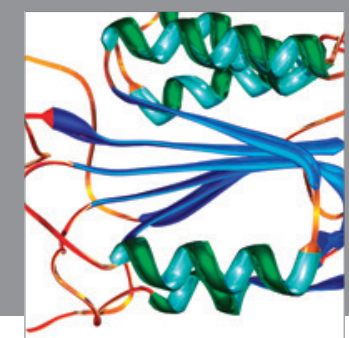

Disease Markers
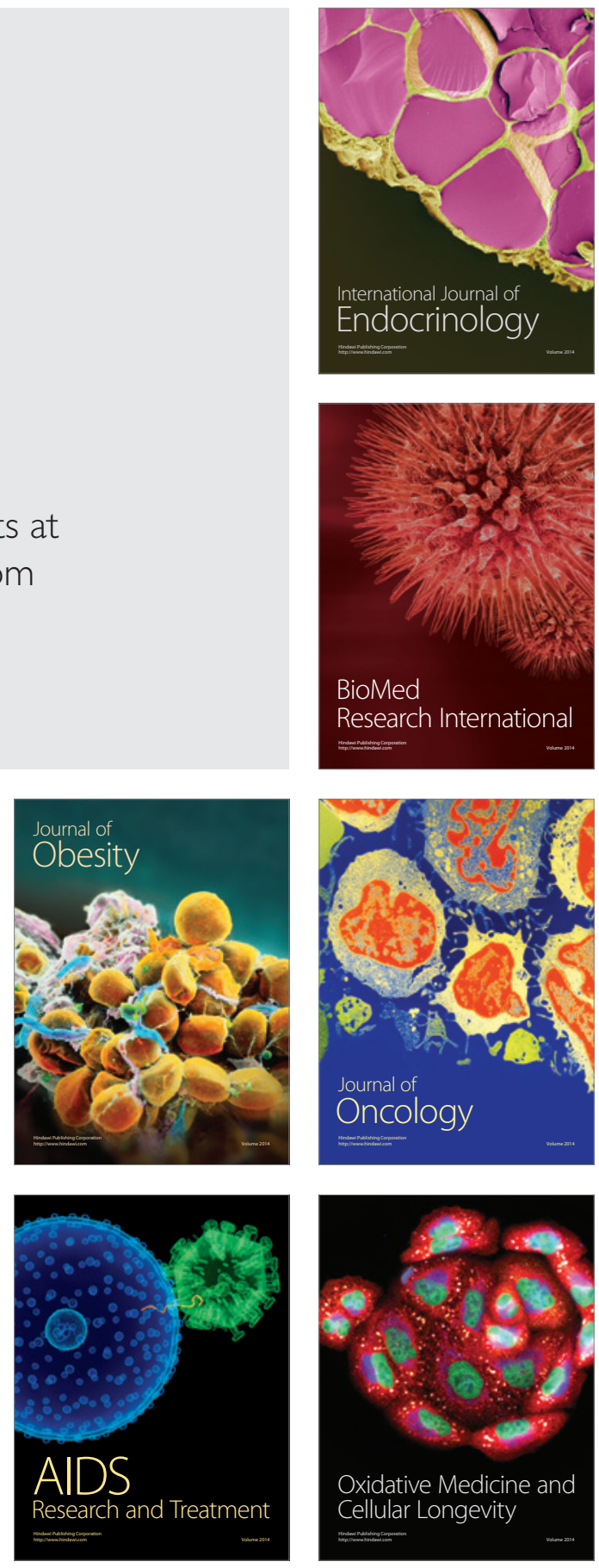